\title{
Hardness Results for Multicast Cost Sharing*
}

\author{
Joan Feigenbaum ${ }^{\dagger}$ Arvind Krishnamurthy ${ }^{\ddagger}$ Rahul Sami ${ }^{\S}$ Scott Shenker ${ }^{\llbracket}$
}

March 9, 2003

\begin{abstract}
We continue the study of multicast cost sharing from the viewpoints of both computational complexity and economic mechanism design. We provide fundamental lower bounds on the network complexity of group-strategyproof, budget-balanced mechanisms. We also extend a classical impossibility result in game theory to show that no strategyproof mechanism can be both approximately efficient and approximately budget-balanced. Our results show that one important and natural case of multicast cost sharing is an example of a canonical hard problem in distributed, algorithmic mechanism design; in this sense, they represent progress toward the development of a complexity theory of Internet computation.
\end{abstract}

Keywords: Algorithmic mechanism design, communication complexity, multicast.

\section{Introduction}

In the standard unicast model of Internet transmission, each packet is sent to a single destination. Although unicast service has great utility and widespread applicability, it cannot efficiently transmit popular content, such as movies or concerts, to a large number of receivers; the source would have to transmit a separate copy of the content to each receiver independently. The multicast model of Internet transmission relieves this problem by setting up a shared delivery tree spanning all the receivers; packets sent down this tree are replicated at branch points so that no more than one copy of each packet traverses each link. Multicast thus greatly reduces the transmission costs involved in reaching large user populations.

\footnotetext{
${ }^{*}$ This work was supported by the DoD University Research Initiative (URI) program administered by the Office of Naval Research under Grant N00014-01-1-0795. It was presented in preliminary form at the 2002 Conference on Foundations of Software Technology and Theoretical Computer Science [FKS+02].

${ }^{\dagger}$ Yale University, Computer Science, New Haven, CT 06520-8285 USA, feigenbaum@cs.yale.edu. Supported in part by ONR grants N00014-01-1-0447 and N00014-01-1-0795 and NSF grants CCR-0105337 and ANI-0207399.

${ }^{\ddagger}$ Yale University, Computer Science, New Haven, CT 06520-8285 USA, arvind@cs.yale.edu. Supported in part by NSF grants CCR-9985304 and ANI-0207399.

${ }^{\S}$ Yale University, Computer Science, New Haven, CT 06520-8285 USA, sami@cs.yale.edu. Supported by ONR grant N00014-01-1-0795.

`ICSI, 1947 Center Street, Berkeley, CA 94704-1198 USA, shenker@icsi.berkeley.edu. Supported in part by NSF grants ANI-9730162, ITR-0081698, ITR-0121555, and ANI-0207399.
} 
The large-scale, high-bandwidth multicast transmissions required for movies and other potential sources of revenue are likely to incur substantial transmission costs. The costs when using the unicast transmission model are separable in that the total cost of the transmission is merely the sum of the costs of transmission to each receiver. Multicast's use of a shared delivery tree greatly reduces the overall transmission costs, but, because the total cost is now a submodular and nonlinear function of the set of receivers, it is not clear how to share the costs among the receivers. A recent series of papers has addressed the problem of cost sharing for Internet multicast transmissions. In the first paper on the topic, Herzog et al considered axiomatic and implementation aspects of the problem. Subsequently, Moulin and Shenker [MS01] studied the problem from a purely economic point of view. Several more recent papers [FPS01, AFK+02, AR02, FGH+02] adopt the distributed algorithmic mechanism design approach, which augments a game-theoretic perspective with distributed computational concerns. In this paper, we extend the results of [FPS01] by considering a more general computational model and approximate solutions. We also extend a classic impossibility [GL79] result by showing that no strategyproof mechanism can be both approximately efficient and approximately budget-balanced.

Before providing a detailed technical statement of our results, we introduce distributed algorithmic mechanism design and our model of multicast cost sharing.

\subsection{Distributed Algorithmic Mechanism Design}

We consider both complexity and incentive issues inherent in multicast cost sharing. This approach is fairly new, as incentives have rarely been an important consideration in traditional theoretical computer science (TCS). Instead, users (or the computers acting on their behalf) are assumed either to be obedient (i.e., to follow the prescribed algorithm) or to be adversaries who "play against" each other. The traditional TCS focus is on the design of computationally efficient algorithms and protocols that accomplish the desired aim in the presence of these obedient or adversarial users.

In contrast, the selfish users in game theory are neither cooperative nor adversarial. Although one cannot assume that selfish users will obediently follow the prescribed algorithm, one can assume that they will respond to incentives. Thus, one need not design algorithms that achieve correct results in the face of adversarial behavior on the part of some users, but one does need algorithms that work correctly in the presence of predictably selfish behavior by all users. Achieving systemwide goals like Pareto efficiency or fairness in the presence of selfish agents is the primary aim of economic mechanism design (see [J01] for a review). However, this mechanism-design literature has not typically considered the algorithmic efficiency of mechanisms.

Thus, while the economics literature traditionally stressed incentives and downplayed computational complexity, the computer-science literature traditionally did the opposite. The emergence of the Internet as a standard platform for distributed computation has changed this state of affairs. Incentives have become an increasingly important consideration in network-protocol design (see, 
for example, [FNY89, FS97, HA88, KLO95, KS89, S88, S90, S95]). More recently, the work of Nisan and Ronen [NR01] has inspired the design of algorithmic mechanisms for, e.g., scheduling, load balancing, lowest-cost paths, and combinatorial auctions that satisfy both the traditional economic definitions of incentive compatibility and the traditional TCS definitions of efficiency. The examples of algorithmic mechanism design in [NR01] use centralized computations and therefore invoke notions of centralized complexity. More recent papers have introduced distributed algorithmic mechanism design (DAMD), in which the computations are distributed and therefore require distributed measures of complexity. Feigenbaum, Papadimitriou, and Shenker [FPS01] propose a general concept of "network complexity" that requires a distributed algorithm executed over an interconnection network $T$ to be modest in four respects: the total number of messages that agents send over $T$, the maximum number of messages sent over any one link in $T$, the maximum size of a message, and the local computational burden on agents. If a distributed algorithm requires an excessive expenditure of any one of these four resources, then its "network complexity" is unacceptable. This notion of network complexity allows the mechanism designer to evaluate the feasibility of executing the algorithmic mechanism in a decentralized setting. Network complexity is not (yet) a precisely defined notion, and further study of distributed algorithmic mechanisms is required to formalize its definition. Each of these four resources has been used as a measure of complexity in the distributed computation literature; however, it is not standard to consider all four resource constraints simultaneously.

A central challenge in the study of algorithmic mechanism design is the search for hard problems and, more generally, the development of a full-fledged "complexity theory" of mechanisms. Superficially, a problem is hard if it cannot be solved in a manner that satisfies both the incentivecompatibility and the computational-tractability requirements. There will be many problems for which this cannot be done; NP-hard problems, for example, cannot be solved in a computationally tractable manner (unless $\mathrm{P}=\mathrm{NP}$ ), and there are no efficient, strategyproof, and budget-balanced solutions to general cost-sharing problems. However, we are not interested in hardness per se but rather in hardness that results from the interplay of incentive compatibility and computational complexity. Thus, a more useful distinction is made by defining a DAMD problem to be a canonical hard problem ${ }^{1}$ if each of these two requirements can be satisfied individually, but they cannot be satisfied simultaneously. Canonical hard problems will help us understand the fundamental nature of hardness in DAMD, as opposed to hardness that results solely from computational issues or solely from incentive issues. When combined with earlier work in, e.g., [MS01, FPS01], the results that we present here show that one important and natural case of multicast cost sharing is a canonical hard problem; to the best of our knowledge, it is the first such example in the literature. Like "network complexity," "canonical hardness" is not (yet) a precisely defined notion; ultimately, one would like to have well defined network complexity classes, one or more natural notions of reduc-

\footnotetext{
${ }^{1}$ In the extended abstract of this paper[FKS +02$]$, these were called "representative hard problems." Here, we use the term "canonical hard problem" in order to be consistent with the DAMD survey paper [FS02].
} 
tion, and canonical hard problems that are complete for some class, but more natural examples of good (and bad) distributed algorithmic mechanisms are needed in order to get to that point. Further discussion of easiness and hardness in DAMD can be found in [FS02].

\section{$1.2 \quad$ Multicast Cost Sharing Model}

We use the multicast-transmission model of [FPS01]: There is a user population $P$ residing at a set of network nodes $N$, which are connected by bidirectional network links $L$. The multicast flow emanates from a source node $\alpha_{s} \in N$; given any set of receivers $R \subseteq P$, the transmission flows through a multicast tree $T(R) \subseteq L$ rooted at $\alpha_{s}$ and spans the nodes at which users in $R$ reside. It is assumed that there is a universal tree $T(P)$ and that, for each subset $R \subseteq P$, the multicast tree $T(R)$ is merely the minimal subtree of $T(P)$ required to reach the elements in $R$. This approach is consistent with the design philosophy embedded in essentially all multicast-routing proposals (see, e.g., [BFC93, DEF+96, HC99, PLB+99]).

Each link $l \in L$ has an associated $\operatorname{cost} c(l) \geq 0$ that is known by the nodes on each end, and each user $i$ assigns a utility value $u_{i}$ to receiving the transmission. Note that $u_{i}$ is known only to user $i$ a priori, and hence user $i$ can strategize by reporting any value $v_{i} \geq 0$ in place of $u_{i}$. A cost-sharing mechanism determines which users receive the multicast transmission and how much each receiver is charged. We let $x_{i} \geq 0$ denote how much user $i$ is charged and $\sigma_{i}$ denote whether user $i$ receives the transmission; $\sigma_{i}=1$ if the user receives the multicast transmission, and $\sigma_{i}=0$ otherwise. We use $u$ to denote the input vector $\left(u_{1}, u_{2}, \ldots, u_{|P|}\right)$. The mechanism $M$ is then a pair of functions $M(u)=(x(u), \sigma(u))$. The practical feasibility of deploying the mechanism on the Internet depends on the network complexity of computing the functions $x(u)$ and $\sigma(u)$. It is important to note that both the inputs and outputs of these functions are distributed throughout the network; that is, each user inputs his $u_{i}$ from his network location, and the outputs $x_{i}(u)$ and $\sigma_{i}(u)$ must be delivered to him at that location.

The receiver set for a given input vector is $R(u)=\left\{i \mid \sigma_{i}=1\right\}$. A user's individual welfare is given by $w_{i}=\sigma_{i} u_{i}-x_{i}$. The cost of the tree $T(R)$ reaching a set of receivers $R$ is $c(T(R))$, and the overall welfare, or net worth, is $N W(R)=u_{R}-c(T(R))$, where $u_{R}=\sum_{i \in R} u_{i}$ and $c(T(R))=\sum_{l \in T(R)} c(l)$. The overall welfare measures the total benefit of providing the multicast transmission (the sum of the utilities minus the total cost).

Our goal is to explore the relationship between incentives and computational complexity, but, before we do so, we first comment on several aspects of the model. The cost model we employ is a poor reflection of reality, in that transmission costs are not per-link; current network-pricing schemes typically only involve usage-based or flat-rate access fees, and the true underlying costs of network usage, though hard to determine, involve small incremental costs (i.e., sending additional packets is essentially free) and large fixed costs (i.e., installing a link is expensive). However, we are not aware of a well-validated alternative cost model, and the per-link cost structure is intuitively appealing, 
relatively tractable, and widely used (e.g., in [AFK+02, AR02, FGH+02, FPS01, JV01, MT02]).

We assume that the total transmission costs are shared among the receivers. There are certainly cases in which the costs would more naturally be borne by the source (e.g., broadcasting an infomercial) or the sharing of costs is not relevant (e.g., a teleconference among participants from the same organization); in such cases, our model would not apply. However, we think that there will be many cases, particularly those involving the widespread dissemination of popular content, in which the costs would be borne by the receivers.

There are certainly cases, such as the high-bandwidth broadcast of a long-lived event such as a concert or movie, in which the bandwidth required by the transmission is much greater than that required by a centralized cost-sharing mechanism (i.e., sending all the link costs and utility values to a central site at which the receiver set and cost shares could be computed). For these cases, our feasibility concerns would be moot. However, Internet protocols are designed to be general-purpose; what we address here is the design of a protocol that would share multicast costs for a wide variety of uses, not just long-lived and high-bandwidth events. Thus, the fact that there are scenarios (e.g., the transmission of a shuttle mission, as explained below) in which our feasibility concerns are relevant is sufficient motivation; they need not be relevant in all scenarios.

In comparing the bandwidth required for transmission to the bandwidth required for the costsharing mechanism, one must consider several factors. First, and most obvious, is the transmission rate $b$ of the application. For large multicast groups, it will be quite likely that there will be at least one user connected to the Internet by a slow modem. Because the multicast rate must be chosen to accommodate the slowest user, one can't assume that $b$ will be large. Second, the bandwidth consumed on any particular link by centralized cost sharing mechanisms scales linearly with the number of users $p=|P|$, but the multicast's usage of the link is independent of the number of users. Third, one must consider the time increment $\Delta$ over which the cost accounting is done. For some events, such as a movie, it would be appropriate to calculate the cost shares once (at the beginning of the transmission) and not allow users to join after the transmission has started. For other events, such as the transmission of a shuttle mission, users would come and go during the course of the transmission. To share costs accurately in such cases, the time increment $\Delta$ must be fairly short. The accounting bandwidth on a single link scales roughly as $p$, which must be compared to the bandwidth $\Delta b$ used over a single accounting interval. Although small multicast groups with large $\Delta$ and $b$ could easily use a centralized mechanism, large multicast groups with small $\Delta$ and $b$ could not.

We have assumed that budget-balanced cost sharing, where the sum of the charges exactly covers the total incurred cost, is the goal of the charging mechanism. If the charging mechanism were being designed by a monopoly network operator, then one might expect the goal to be maximizing revenue. There have been some recent investigations of revenue-maximizing charging schemes for multicast (see, e.g., [FGH+02]), but here we assume, as in [HSE97, MS01, FPS01, AFK+02, AR02], that the charging mechanism is decided by society at large (e.g., through standards bodies) or 
through competition. Competing network providers could not charge more than their real costs (or otherwise their prices would be undercut) nor less than their real costs (or else they would lose money), and so budget balance is a reasonable goal in such a case. For some applications, such as big-budget movies, the bandwidth costs will be insignificant compared to the cost of the content, and then different charging schemes will be needed, but for low-budget or free content (e.g., teleconferences) budget-balanced cost-sharing is appropriate.

Lastly, in our model it is the users who are selfish. The routers (represented by tree nodes), links, and other network-infrastructure components are obedient. Thus, the cost-sharing algorithm does not know the individual utilities $u_{i}$, and so users could lie about them, but once they report them to the network infrastructure (e.g., by sending them to the nearest router or accounting node), the algorithms for computing $x(u)$ and $\sigma(u)$ can be reliably executed by the network. Ours is the simplest possible strategic model for the distributed algorithmic mechanism-design problem of multicast cost sharing, but, even in this simplest case, determining the inherent network complexity of the problem is non-trivial. Alternative strategic models (e.g., ones in which the routers are selfish, and their strategic goals may be aligned or at odds with those of their resident users) may also present interesting distributed algorithmic mechanism-design challenges. Preliminary work along these lines is reported in [MT02]. Finally, we note that our framework of distributed algorithmic mechanism design includes both distributed information (inputs and outputs) and distributed computation; there has also been work on studying the impact of distributed information alone on algorithmic mechanism design [MT99, NS02].

\subsection{Statement of Results}

In order to state our results more precisely, we need additional notation and terminology.

A strategyproof cost-sharing mechanism is one that satisfies the property that $w_{i}(u) \geq w_{i}\left(\left.u\right|^{i} \mu_{i}\right)$, for all $u, i$, and $\mu_{i}$. (Here, $\left(\left.u\right|^{i} \mu_{i}\right)_{j}=u_{j}$, for $j \neq i$, and $\left(\left.u\right|^{i} \mu_{i}\right)_{i}=\mu_{i}$. In other words, $\left.u\right|^{i} \mu_{i}$ is the utility profile obtained by replacing $u_{i}$ by $\mu_{i}$ in $u$.) Strategyproofness does not preclude the possibility of a group of users colluding to improve their individual welfares. Any reported utility profile $v$ can be considered a group strategy for any group $S \supseteq\left\{i \mid v_{i} \neq u_{i}\right\}$. A mechanism $M$ is group-strategyproof (GSP) if there is no group strategy such that at least one member of the strategizing group improves his welfare while the rest of the members do not reduce their welfare. In other words, if $M$ is GSP, the following property holds for all $u, v$, and $S \supseteq\left\{i \mid u_{i} \neq v_{i}\right\}$ :

$$
\begin{gathered}
\text { either } w_{i}(v)=w_{i}(u), \forall i \in S, \\
\text { or } \exists i \in S \text { such that } w_{i}(v)<w_{i}(u) .
\end{gathered}
$$

In general, we only consider mechanisms that satisfy four natural requirements ${ }^{2}$ :

\footnotetext{
${ }^{2}$ The one exception is Section 4, in which we do not assume SYM; that section contains an impossibility result, and so not making this assumption only makes the section stronger.
} 
No Positive Transfers (NPT): $x_{i}(u) \geq 0$; in other words, the mechanism cannot pay receivers to receive the transmission.

Voluntary Participation (VP): $w_{i}(u) \geq 0$; this implies that users are not charged if they do not receive the transmission and that users who do receive the transmission are not charged more than their reported utilities.

Consumer Sovereignty (CS): For given $T(P)^{3}$ and link costs $c(\cdot)$, there exists some $\mu_{c s}$ such that $\sigma_{i}(u)=1$ if $u_{i} \geq \mu_{c s}$; this condition ensures that the network cannot exclude any agent who is willing to pay a sufficiently large amount, regardless of other agents' utilities.

Symmetry $^{4}$ (SYM): If $i$ and $j$ are at the same node or are at different nodes separated by a zero-cost path, and $u_{i}=u_{j}$, then $\sigma_{i}=\sigma_{j}$ and $x_{i}=x_{j}$.

In addition to these basic requirements, there are certain other desirable properties that one could expect a cost-sharing mechanism to possess. A cost-sharing mechanism is said to be efficient if it maximizes the overall welfare, and it is said to be budget-balanced if the revenue raised from the receivers covers the cost of the transmission exactly. It is a classical result in game theory [GL79] that a strategyproof cost-sharing mechanism that satisfies NPT, VP, and CS cannot be both budgetbalanced and efficient. Moulin and Shenker [MS01] have shown that there is only one strategyproof, efficient mechanism, called marginal cost (MC), defined in Section 4 below, that satisfies NPT, VP, and CS. They have also shown that, while there are many GSP, budget-balanced mechanisms that satisfy NPT, VP, and CS, the most natural one to consider is the Shapley value (SH), defined in Section 2 below, because it minimizes the worst-case efficiency loss.

Both MC and SH also satisfy the SYM property. The egalitarian (EG) mechanism of Dutta and Ray [DR89] is another well studied GSP, budget-balanced mechanism that satisfies the four basic requirements. Jain and Vazirani [JV01] present a novel family of GSP, approximately budgetbalanced mechanisms ${ }^{5}$ that satisfy NPT, VP, and CS. Each mechanism in the family is defined by its underlying cost-sharing function, and the resulting mechanism satisfies the SYM property whenever the underlying function satisfies it. We use the notation JV to refer to the members of the Jain-Vazirani family that satisfy SYM.

It is noted in [FPS01] that, for multicast cost sharing, both $\mathrm{MC}$ and $\mathrm{SH}$ are polynomial-time computable by centralized algorithms. Furthermore, there is a distributed algorithm given in [FPS01] that computes MC using only two short messages per link and two simple calculations per node. By contrast, [FPS01] notes that the obvious algorithm that computes SH requires $\Omega(|P| \cdot|N|)$ messages in the worst case and shows that, for a restricted class of algorithms (called "linear distributed algorithms"), there is an infinite set of instances with $|P|=O(|N|)$ that require $\Omega\left(|N|^{2}\right)$

\footnotetext{
${ }^{3}$ For brevity, we often use $T(P)$ to denote four components of a multicast cost-sharing problem instance: the node-set $N$, the link-set $L$, the locations of the agents, and the multicast-source location $\alpha_{s}$.

${ }^{4}$ This straightforward definition is less restrictive than the one given by Moulin and Shenker [MS01]. The SH, JV, and EG mechanisms that we use as examples satisfy the more stringent definition of symmetry in [MS01] as well.

${ }^{5}$ The mechanisms in [JV01] actually satisfy a more stringent definition of approximate budget balance than we use; thus, our network-complexity lower bounds apply to them a fortiori.
} 
messages. Jain and Vazirani [JV01] give centralized, polynomial-time algorithms to compute the approximately budget-balanced mechanisms in the class JV.

In this paper, we show that:

- Any distributed algorithm, deterministic or randomized, that computes a budget-balanced, GSP multicast cost-sharing mechanism must send $\Omega(|P|)$ bits over linearly many links in the worst case. This lower bound applies, in particular, to the SH and EG mechanisms.

- Any distributed algorithm, deterministic or randomized, that computes an approximately budget-balanced, GSP multicast cost-sharing mechanism must send $\Omega(\log (|P|))$ bits over linearly many links in the worst case. This lower bound applies, in particular, to the SH, EG, and JV mechanisms.

(In both these results, the "worst case" is worst with respect to all possible network topologies, link costs, and user utilities.)

In order to prove the first of these lower bounds (i.e., the one for exact budget balance), we first prove a lower bound that holds for all mechanisms that correspond to strictly cross-monotonic cost-sharing functions. Cross-monotonicity, a technical property defined precisely in Section 2, means roughly that the cost share attributed to any particular receiver cannot increase as the receiver set grows; the $\mathrm{SH}$ and EG cost-sharing functions for a broad class of multicast trees are examples of strictly cross-monotonic functions but not the only examples. Our lower bound on the network complexity of strictly cross-monotonic mechanisms may be applicable to problems other than multicast.

It is well known that there is no strategyproof mechanism that is both (exactly) efficient and budget-balanced on all problem instances [GL79]. This in itself does not rule out the existence of a strategyproof mechanism that is approximately efficient and approximately budget-balanced. However, we prove that this is also impossible:

- There is no strategyproof multicast cost-sharing mechanism satisfying NPT, VP, and CS that is both approximately efficient and approximately budget-balanced.

\section{$2 \quad$ Exact submodular cost sharing}

In this section, we prove a basic communication-complexity lower bound that applies to the distributed computation of many submodular cost-sharing mechanisms. We first prove this lower bound for all mechanisms that satisfy "strict cross-monotonicity" as well as the four basic properties discussed in Section 1. We then show that, whenever the underlying cost function is strictly subadditive, the resulting Shapley-value mechanism is strictly cross-monotonic and hence has poor network complexity. Finally, we discuss the special case of multicast cost sharing and describe very general conditions under which the multicast cost will be strictly subadditive. In particular, we 
present an infinite family of instances that have strictly subadditive costs and show that, on these instances, any cost-sharing mechanism that satisfies the four basic requirements is equivalent to $\mathrm{SH}$ and must have poor network complexity.

Consider the general situation in which we want a mechanism to allow the users to share the cost of a common service. We restrict our attention to the case of binary preferences: User $i$ is either "included," by which he attains utility $u_{i}$, or he is "excluded" from the service, giving him 0 utility. A mechanism can use the utility vector $u$ as input to compute a set $R(u)$ of users who receive the service and a payment vector $x(u)$. Further, suppose that the cost of serving a set $S \subseteq P$ of the users is given by $C(S)$. This cost function is called submodular if, for all $S, T \subseteq P$, it satisfies:

$$
C(S \cup T)+C(S \cap T) \leq C(S)+C(T) .
$$

Submodularity is often used to model economies of scale, in which the marginal costs decrease as the serviced set grows. One example of a submodular cost function is the one presented in Section 1 , where the cost of delivering a multicast to a set $R$ of receivers is the sum of the link costs in the smallest subtree of the universal tree that includes all locations of users in $R$.

Moulin and Shenker [M99, MS01] have shown that any mechanism for submodular cost sharing that satisfies budget-balance, GSP, VP, and NPT must belong to the class of cross-monotonic costsharing mechanisms. A mechanism in this class is completely characterized by its set of cost-sharing functions $g=\left\{g_{i}: 2^{P} \rightarrow \Re_{\geq 0}\right\}$. Here $g_{i}(S)$ is the cost that $g$ attributes to user $i$ if the receiver set is $S$. For brevity, we will refer to $g=\left\{g_{i}\right\}$ as a "cost-sharing function," rather than a set of cost-sharing functions. We say that $g$ is cross-monotonic if, $\forall i \in S, \forall T \subseteq P, g_{i}(S \cup T) \leq g_{i}(S)$. In addition, we require that $g_{i}(S) \geq 0$ and, $\forall j \notin S, g_{j}(S)=0$. Then, the corresponding crossmonotonic mechanism $M_{g}=(\sigma(u), x(u))$ is defined as follows: The receiver set $R(u)$ is the unique largest set $S$ for which $g_{i}(S) \leq u_{i}$, for all $i$. This is well defined, because, if sets $S$ and $T$ each satisfy this property, then cross-monotonicity implies that $S \cup T$ satisfies it. The cost shares are then set at $x_{i}(u)=g_{i}(R(u))$.

There is a natural iterative algorithm to compute a cross-monotonic cost-sharing mechanism [MS01, FPS01]: Start by assuming the receiver set $R^{0}=P$, and compute the resulting cost shares $x_{i}^{0}=g_{i}\left(R^{0}\right)$. Then drop out any user $j$ such that $u_{j}<x_{j}^{0}$; call the set of remaining users $R^{1}$. The cost shares of other users may have increased, and so we need to compute the new cost shares $x_{i}^{1}=g_{i}\left(R^{1}\right)$ and iterate. This process ultimately converges, terminating with the receiver set $R(u)$ and the final cost shares $x_{i}(u)$.

Now, we consider a subclass of the cross-monotonic mechanisms:

Definition 1 A cross-monotonic cost-sharing function $g=\left\{g_{i}: 2^{P} \rightarrow \Re_{\geq 0}\right\}$ is called strictly cross-monotonic if, for all $S \subset P, i \in S$, and $j \notin S, g_{i}(S \cup\{j\})<g_{i}(S)$. The corresponding mechanism $M_{g}$ is called a strictly cross-monotonic mechanism.

We now prove a lower bound on the communication complexity of strictly cross-monotonic 


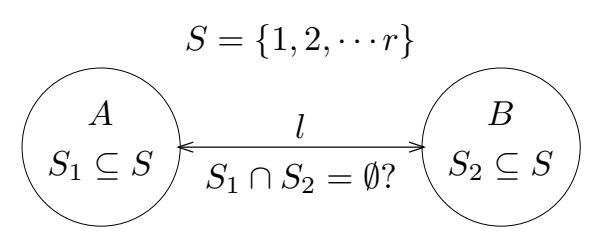

Figure 1: The set disjointness problem

cost-sharing mechanisms. Our proof is a reduction from the set disjointness problem: Consider a network consisting of two nodes $A$ and $B$, separated by a link $l$ (see Figure 1). Node $A$ has a set $S_{1} \subseteq\{1,2, \ldots, r\}$, node $B$ has another set $S_{2} \subseteq\{1,2, \ldots, r\}$, and one must determine whether the sets $S_{1}$ and $S_{2}$ are disjoint. It is known that any deterministic or randomized algorithm to solve this problem must send $\Omega(r)$ bits between $A$ and $B$. (Proofs of this and other basic results in communication complexity can be found in [KN97].)

Theorem 1 Suppose $M_{g}$ is a strictly cross-monotonic mechanism corresponding to a cost-sharing function $g$ and satisfying VP, CS, and NPT. Further, suppose that the mechanism must be computed in a network in which a link (or set of links) $l$ is a cut and there are $\Omega(|P|)$ users on each side of $l$. Then, any deterministic or randomized algorithm to compute $M_{g}$ must send $\Omega(|P|)$ bits across $l$ in the worst case.

Proof: For simplicity, assume that the network consists of two nodes $A$ and $B$ connected by one link $l$ and that there are $r=|P| / 2$ users at each of the two nodes. (The proof of the more general case is identical.) Arbitrarily order the users at each node. We can now call the users $a_{1}, a_{2}, \ldots, a_{r}$ and $b_{1}, b_{2}, \ldots, b_{r}$. Because the mechanism $M_{g}$ is strictly cross-monotonic, we can find a real value $d>0$ such that, for all $S \subset P, i \in S, j \notin S$,

$$
g_{i}(S \cup\{j\})<g_{i}(S)-d .
$$

For each user $i \in P$, we will define two possible utility values $t_{i}^{L}$ and $t_{i}^{H}$ as follows:

- First, the values for $a_{1}$ and $b_{1}$ are

$$
\begin{array}{ll}
t_{a_{1}}^{H}=g_{a_{1}}\left(\left\{a_{1}, b_{1}\right\}\right), & t_{a_{1}}^{L}=t_{a_{1}}^{H}-d \\
t_{b_{1}}^{H}=g_{b_{1}}\left(\left\{a_{1}, b_{1}\right\}\right), & t_{b_{1}}^{L}=t_{b_{1}}^{H}-d
\end{array}
$$

- Similarly, the values for $a_{k}$ and $b_{k}$ are

$$
\begin{array}{ll}
t_{a_{k}}^{H}=g_{a_{k}}\left(\left\{a_{1}, a_{2}, \ldots, a_{k}, b_{1}, b_{2}, \ldots, b_{k}\right\}\right), & t_{a_{k}}^{L}=t_{a_{k}}^{H}-d \\
t_{b_{k}}^{H}=g_{b_{k}}\left(\left\{a_{1}, a_{2}, \ldots, a_{k}, b_{1}, b_{2}, \ldots, b_{k}\right\}\right), & t_{b_{k}}^{L}=t_{b_{k}}^{H}-d
\end{array}
$$


Now, we show how to reduce from the set disjointness problem to the mechanism $M_{g}$. Node $A$ gets a subset $S_{1} \subseteq\{1, \ldots, r\}$ and constructs a utility vector $u$ for the users at $A$, defined by

$$
\begin{aligned}
& \forall i \in S_{1} \quad u_{a_{i}}=t_{a_{i}}^{H} \\
& \forall i \notin S_{1} \quad u_{a_{i}}=t_{a_{i}}^{L}
\end{aligned}
$$

Similarly, node $B$ is given set $S_{2}$ and constructs a utility vector $v$ for the users at $B$, defined by

$$
\begin{aligned}
& \forall i \in S_{2} \quad v_{b_{i}}=t_{b_{i}}^{H} \\
& \forall i \notin S_{2} \quad v_{b_{i}}=t_{b_{i}}^{L}
\end{aligned}
$$

They now run mechanism $M_{g}$ on input $(u, v)$ and check whether the receiver set $R_{g}(u, v)$ is empty.

Claim: $R_{g}(u, v)$ is empty iff $S_{1}$ and $S_{2}$ are disjoint.

Proof of claim: To show the "if" direction, we can simulate the iterative algorithm to compute the receiver set. We start with $R=P$. Then, because $S_{1}$ and $S_{2}$ are disjoint, either $r \notin S_{1}$ or $r \notin S_{2}$. Assume, without loss of generality, that $r \notin S_{1}$. Now, $u_{a_{r}}=t_{a_{r}}^{L}<g_{a_{r}}(R)$, and hence $a_{r}$ must drop out of the receiver set $R$. But now, because of strict cross-monotonicity, it follows that $g_{b_{r}}\left(P-\left\{a_{r}\right\}\right)>g_{b_{r}}(P)=t_{b_{r}}^{H}$, and so $b_{r}$ must also drop out of the receiver set. Repeating this argument for $r-1, r-2, \ldots, 1$, we can show that the receiver set must be empty.

To show the "only if" direction, assume that $i \in S_{1} \cap S_{2}$. Then, let $T=\left\{a_{1}, \ldots, a_{i}, b_{1}, \ldots, b_{i}\right\}$. $u_{a_{i}}=t_{a_{i}}^{H}=g_{a_{i}}(T)$, and $v_{a_{i}}=t_{b_{i}}^{H}=g_{b_{i}}(T)$. Further, for all $j<i$, it follows from strict crossmonotonicity that $g_{a_{j}}(T)<t_{a_{j}}^{L} \leq u_{a_{j}}$, and $g_{b_{j}}(T)<t_{b_{j}}^{L} \leq v_{b_{j}}$. Thus, the receiver set $R_{g}(u, v) \supseteq T$, and hence it is nonempty.

Theorem 1 follows from this claim and the communication complexity of set disjointness.

\subsection{Strictly Subadditive Cost Functions}

In this section, we show that, for a class of submodular cost functions, the Shapley-value mechanism (which is perhaps the most compelling mechanism from an economic point of view) is strictly crossmonotonic and hence has poor network complexity.

Theorem 1 provides a sufficient condition, strict cross-monotonicity, for a mechanism to have poor network complexity. However, for some submodular cost functions, it is possible that no mechanism satisfies this condition: If the costs are additive, i.e., if the cost of serving a set $S$ is exactly the sum of the costs of serving each of its members individually, then there is a unique mechanism satisfying the basic properties. This mechanism is defined by:

$$
\begin{gathered}
R(u)=\left\{i \mid u_{i} \geq C(\{i\})\right\} \\
x_{i}(u)=C(\{i\}) \text { if } i \in R(u) \text {, and } x_{i}(u)=0 \text { otherwise. }
\end{gathered}
$$


This mechanism is very easy to compute, either centrally or in a distributed manner, because there is no interaction among the users' utilities; in essence, we have $|P|$ independent local computations to perform.

We need to exclude these trivial cost functions in order to prove general lower bounds for a class of submodular functions. This leads us to consider submodular cost functions that are strictly subadditive:

$$
\forall S \subseteq P, S \neq \emptyset, \quad \forall i \in P, \quad C(S \cup\{i\})<C(S)+C(\{i\})
$$

For a given cost function $C$, there may be many $g=\left\{g_{i}\right\}$ for which the corresponding mechanism $M_{g}$ satisfies the basic properties NPT, VP, CS, and SYM. However, Moulin and Shenker [M99, MS01] have shown that, for any given submodular cost function, the cross-monotonic mechanism that minimizes the worst-case efficiency loss is the Shapley-value mechanism (SH). This is a crossmonotonic cost-sharing mechanism corresponding to a function $g^{S H}$, defined by:

$$
\forall S \subseteq P \quad \forall i \in S, \quad g_{i}^{S H}(S)=\sum_{R \subseteq S-\{i\}} \frac{|R| !(|S|-|R|-1) !}{|S| !}[C(R \cup\{i\})-C(R)]
$$

The SH mechanism is therefore a natural mechanism to choose for submodular cost sharing. The following lemma shows that this mechanism has poor network complexity.

Lemma 1 The SH mechanism for a strictly subadditive cost function is strictly cross-monotonic.

Proof: We need to show that, for all sets $S$, for all $i \in S, j \notin S, g_{i}^{S H}(S \cup\{j\})<g_{i}^{S H}(S)$. The proof follows directly from the definition of $g_{i}^{S H}(S)$ in Equation 1. We use $\mathrm{MC}_{i}(R)$ to denote $[C(R \cup\{i\})-C(R)]$, the marginal cost of serving $i$ in set $R$. Consider a set $S \subseteq P-\{j\}$ and a user $i \in S$. Let $r=|R|, s=|S|$.

$$
\begin{aligned}
g_{i}^{S H}(S \cup\{j\}) & =\sum_{R \subseteq S \cup\{j\}-\{i\}} \frac{r !(s-r) !}{(s+1) !} \mathrm{MC}_{i}(R) \\
& =\sum_{R \subseteq S-\{i\}}\left[\frac{r !(s-r) !}{(s+1) !} \mathrm{MC}_{i}(R)+\frac{(r+1) !(s-r-1) !}{(s+1) !} \mathrm{MC}_{i}(R \cup\{j\})\right] \\
& =\sum_{R \subseteq S-\{i\}} \frac{r !(s-r-1) !}{s !}\left[\frac{s-r}{s+1} \mathrm{MC}_{i}(R)+\frac{r+1}{s+1} \mathrm{MC}_{i}(R \cup\{j\})\right]
\end{aligned}
$$

It follows from submodularity of costs that, for all $R, \mathrm{MC}_{i}(R \cup\{j\}) \leq \mathrm{MC}_{i}(R)$. Further, strict subadditivity implies that, for $R=\emptyset, \mathrm{MC}_{i}(R \cup\{j\})<\mathrm{MC}_{i}(R)$. Thus, Equation 2 yields

$$
\begin{aligned}
g_{i}^{S H}(S \cup\{j\}) & <\sum_{R \subseteq S-\{i\}} \frac{r !(s-r-1) !}{s !} \mathrm{MC}_{i}(R) \\
g_{i}^{S H}(S \cup\{j\}) & <g_{i}^{S H}(S)
\end{aligned}
$$


Corollary 1 For a strictly subadditive cost function, any algorithm (deterministic or randomized) that computes the SH mechanism in a network must communicate $\Omega(|P|)$ bits across any cut that has $\Theta(|P|)$ users on each side of the cut.

Note that the network may consist of a root node $\alpha_{s}$ with no resident users, a node $A$ with $\frac{|P|}{2}$ resident users, another node $B$ with $\frac{|P|}{2}$ resident users, a link from $\alpha_{s}$ to $A$, and a path from $A$ to $B$ consisting of $|N|-3$ nodes, each with no resident users. Each link in the path from $A$ to $B$ is a cut with $\Theta(|P|)$ users on each side, and thus $\Omega(|P|)$ bits must be sent across linearly many links. In what follows, we call these the path instances.

\section{$2.2 \quad$ Multicast cost sharing}

We now return to the special case of multicast cost sharing. Recall that the cost function associated with an instance of the multicast cost-sharing problem is determined by the structure of the universal multicast tree $T$, the link costs, and the locations of the users in the tree; so the cost $C(S)$ of serving user set $S \subseteq P$ is $\sum_{l \in T(S)} c(l)$, where $T(S)$ is the smallest subtree of $T$ that includes all nodes at which users in $S$ reside. It is not hard to show that there are many instances that give rise to strictly subadditive functions $C$. In fact, we have the following lemma:

Lemma 2 Consider any instance of multicast cost sharing in which, for every two potential receivers $i$ and $j$, there exists a link $l \in T(\{i\}) \cap T(\{j\})$ such that $c(l)>0$. The cost function associated with this instance is strictly subadditive.

Proof: Given $S \subseteq P-i$, pick any $j \in S$. Let $l$ be any link in $T(\{j\}) \cap T(\{i\})$ with $c(l)>0$. Then, $C(S \cup\{i\}) \leq C(S)+C(\{i\})-c(l)<C(S)+C(\{i\})$.

For example, whenever the source of the multicast has only one link from it, and this link has non-zero cost, the associated cost function is strictly subadditive. One such family of instances (parametrized by $p=|P|$ ) is shown in Figure 2. There are three nodes, $\alpha_{s}, A$, and $B$, and $p$ users. There are $(p / 2)$ users at each of $B$ and $A$, with utilities $u_{1}, u_{2}, \ldots, u \frac{p}{2}$ and $v_{1}, v_{2}, \ldots, v_{\frac{p}{2}}$, respectively. The link $l_{1}$ between $\alpha_{s}$ and $A \operatorname{costs} C$, while the link $l_{2}$ between $A$ and $B$ is free (has 0 cost).

It follows immediately from Corollary 1 that the Shapley-value mechanism for this family of trees requires $\Omega(|P|)$ bits of communication across linearly many links. In addition, we now show that any mechanism that satisfies the basic properties, such as EG, must be identical to the SH mechanism on instances of the type shown in Figure 2; thus, the lower bound extends to all such mechanisms.

Lemma 3 Consider multicast cost-sharing instances of the type shown in Figure 2. Let $M_{g}$ be a cross-monotonic cost-sharing mechanism that satisfies SYM, corresponding to a cost-sharing function $g=\left\{g_{i}\right\}$. Then, $g$ (and $M_{g}$ ) are completely determined on these instances by

$$
\forall S \subseteq P, \forall i \in S \quad g_{i}(S)=\frac{C}{|S|} .
$$




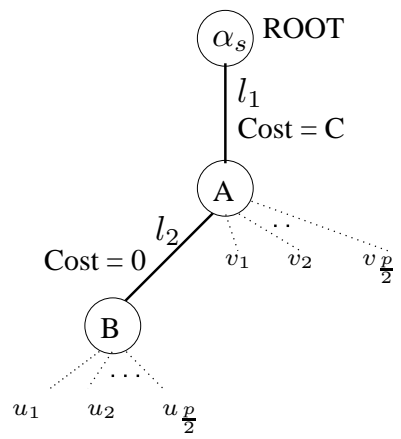

Figure 2: Multicast tree with strictly subadditive costs

Proof: For any receiver set $S$, if the utility values of all users in $S$ are increased to some sufficiently large value, the receiver set will still be $S$. Because the mechanism satisfies SYM, this implies that $g_{i}(S)=g_{j}(S)$ for any pair $i, j$ in this set. The budget-balance requirement then forces $g_{i}(S)=\frac{C}{|S|}$ for all $i$ in set $S$.

It follows from Lemma 3 that any such mechanism must be strictly cross-monotonic on this family of instances. Thus, Theorem 1 and Lemma 3 imply the following lower bound for multicast cost sharing. The worst-case instances include the path instances defined in Section 2.1, with cost $C$ on the link from $\alpha_{s}$ to $A$ and cost 0 on all the other links; these instances are identical to the ones in Figure 2, except that they contain a zero-cost path of length $|N|-2$ from $A$ to $B$ instead of a single zero-cost link.

Theorem 2 Any distributed algorithm, deterministic or randomized, that exactly computes a budgetbalanced, GSP multicast cost-sharing mechanism that satisfies the four basic properties must send $\Omega(|P|)$ bits over linearly many links in the worst case.

Note that this lower bound applies to the EG mechanism for multicast cost-sharing referred in Section 1.

\section{Network complexity of approximately budget-balanced mecha- nisms}

In view of the lower bounds presented in Section 2, it is natural to ask whether one can approximate a budget-balanced, GSP mechanism in a communication-efficient manner. In this case, we do not have a clean analogue of Corollary 1 , because cross-monotonic cost functions no longer characterize the class of feasible mechanisms. However, for the special case of multicast cost sharing, we can still prove a result similar to Theorem 2 that provides a lower bound on the network complexity of approximately budget-balanced, GSP mechanisms. 
First, we recall the definition of an "approximately budget-balanced" mechanism. As explained at length in, e.g., [NR00, AFK+02], one cannot define an approximation of a cost-sharing mechanism $(\sigma, x)$ simply as a pair $\left(\sigma^{\prime}, x^{\prime}\right)$ such that $\sigma^{\prime}$ and $x^{\prime}$ approximate $\sigma$ and $x$, respectively, as functions. Such an approach may destroy the game-theoretic properties of $(\sigma, x)$, e.g., the resulting "mechanism" $\left(\sigma^{\prime}, x^{\prime}\right)$ may not be strategyproof! For our purposes in this section, a $\kappa$-approximately budget-balanced mechanism, where $\kappa>1$ is a constant, is a mechanism $(\sigma, x)$ with the following properties: VP, NPT, CS, SYM, and

$$
\forall c(\cdot), T(P), \text { and } u:(1 / \kappa) \cdot c(T(R(u))) \leq \sum_{i \in R(u)} x_{i}(u) \leq \kappa \cdot c(T(R(u))) .
$$

An approximation to a specific budget-balanced mechanism such as $\mathrm{SH}$ or EG would have to satisfy at least one additional (non-strategic) condition. For example, because SH is the GSP, budget-balanced mechanism that minimizes worst-case efficiency loss, an approximation to $\mathrm{SH}$ would have to come within a constant factor of SH's efficiency loss in the worst case.

We extend the lower-bound technique of the previous section so that it applies to $\kappa$-approximately budget-balanced mechanisms, when $\kappa$ is upper-bounded away from $\sqrt{2}$, i.e., when $\kappa \leq \sqrt{2}-\epsilon$, for some fixed $\epsilon>0$. As before, we want to reduce from the set-disjointness problem where node $A$ has a set $S_{1} \subseteq\{1,2, \ldots, r\}$, node $B$ has another set $S_{2} \subseteq\{1,2, \ldots, r\}$, and one must determine whether the sets $S_{1}$ and $S_{2}$ are disjoint. We again construct the multicast tree shown in Figure 2 with $(p / 2)$ users at each of $B$ and $A$, with utilities $u_{1}, u_{2}, \ldots, u_{\frac{p}{2}}$ and $v_{1}, v_{2}, \ldots, v_{\frac{p}{2}}$, respectively.

We first prove some basic lemmas about group-strategyproof mechanisms for this multicast cost sharing problem.

Lemma 4 Let $M$ be a $\kappa$-approximately budget-balanced mechanism for the multicast cost sharing problem in Figure 2 that satisfies GSP. Then, if $\mu$ is a utility profile of the $p$ users such that

$$
\exists h \geq 1 \text { such that } \forall i \in\{1,2, \ldots, h\} \quad \mu_{i}>\kappa C / h
$$

then the receiver set $R(\mu)$ specified by this mechanism is nonempty.

Proof: Let $\mu$ be such a utility profile, and consider any value of $h$ for which the given condition holds. Let $\mu_{c s}$ be the bound for which the CS condition holds, i.e., if $\mu_{i} \geq \mu_{c s} \Rightarrow i \in R(\mu)$. Let $S=\{1,2, \ldots, h\}$. Define a utility profile $\mu^{S}$ by

$$
\begin{aligned}
\mu_{i}^{S} & =\mu_{c s} \quad \forall i \in S \\
\mu_{i}^{S} & =\mu_{i} \quad \forall i \notin S
\end{aligned}
$$

By the CS condition, $S \subseteq R\left(\mu^{S}\right)$. Further, by the SYM condition, we must have $\forall i, j \in$ $S, \quad x_{i}\left(\mu^{S}\right)=x_{j}\left(\mu^{S}\right)$. Further, because the NPT condition implies that $x_{i}\left(\mu^{S}\right) \geq 0$, for all $i \notin S$, and the approximate budget-balance condition requires that the revenue be bounded by $\kappa C$, we must have $x_{i}\left(\mu^{S}\right) \leq \kappa C / h$, for all $i \in S$. 
It follows that $\forall i \in S, x_{i}\left(\mu^{S}\right)<\mu_{i}$. Now suppose that $R(\mu)$ is empty. Then, at utility profile $\mu$, the coalition $S$ could strategize to report a utility profile $\mu^{S}$; then, for each $i \in S, i$ would receive the transmission and pay less than $\mu_{i}$ for it. This would constitute a successful group strategy, which contradicts the assumption that $M$ is group-strategyproof.

Lemma 5 Let $M$ be a $\kappa$-approximately budget-balanced mechanism for the multicast cost sharing problem in Figure 2 that satisfies GSP. Then, if $\mu$ is a utility profile such that

$$
\begin{gathered}
\mu_{1} \geq \mu_{2} \geq \ldots \geq \mu_{p} \quad \text { and } \\
\nexists h \geq 1 \text { such that } \mu_{h} \geq C /(\kappa h)
\end{gathered}
$$

then the receiver set $R(\mu)$ specified by this mechanism is empty.

Proof: Let $\mu$ be such a utility profile, and let $S=R(\mu)$. Suppose that $S \neq \emptyset$. Let $h=$ $\max \{i \mid i \in S\}$, which implies that $\forall i \in S, \mu_{i} \geq \mu_{h}$. By the conditions of the lemma, $\mu_{h}<C /(\kappa h)$; thus, the approximate budget-balance condition combined with VP implies that $\exists j \in S$ such that $x_{j}(\mu)>x_{h}(\mu)$. It then follows that $\mu_{j}>\mu_{h}$. (If $\mu_{j}=\mu_{h}$, then by SYM we would have $x_{j}(\mu)=x_{h}(\mu)$.)

Now, define the utility profile $\mu^{\prime}$ by

$$
\begin{aligned}
& \mu_{h}^{\prime}=\mu_{j} \\
& \mu_{i}^{\prime}=\mu_{i} \quad \forall i \neq h
\end{aligned}
$$

If $h \notin R\left(\mu^{\prime}\right)$, then at utility profile $\mu^{\prime}, h$ could strategize to report $\mu_{h}$ and get transmission with payment $x_{h}(\mu)$; this would be a successful strategy because $x_{h}(\mu)<x_{j}(\mu) \leq \mu_{j}=\mu_{h}^{\prime}$. Mechanism $\mathrm{M}$ is strategyproof, so we must have $h \in R\left(\mu^{\prime}\right)$. Further, we must also have $x_{h}\left(\mu^{\prime}\right)=x_{h}(\mu)$ for the same reason: If $x_{h}\left(\mu^{\prime}\right)>x_{h}(\mu)$, then $h$ could strategize at $\mu^{\prime}$, and, if $x_{h}(\mu)>x_{h}\left(\mu^{\prime}\right)$, then $h$ could strategize at $\mu$.

Now, by applying the SYM condition at $\mu^{\prime}$, we must have $j \in R\left(\mu^{\prime}\right)$ and $x_{j}\left(\mu^{\prime}\right)=x_{h}\left(\mu^{\prime}\right)$. This implies that $x_{j}\left(\mu^{\prime}\right)=x_{h}(\mu)<x_{j}(\mu)$. But now, $h$ and $j$ could collude and strategize at $\mu$ to report $\mu^{\prime}$; this strictly increases $j$ 's welfare (as her payment is strictly reduced), and leaves $h$ 's welfare unchanged, and hence it would be a successful group strategy. This contradicts the fact that $M$ satisfies GSP.

The ordering condition $\mu_{1} \geq \mu_{2} \geq \ldots \geq \mu_{p}$ in Lemma 5 is only included for simplicity of the exposition; we can always relabel the agents such that it holds.

Theorem 3 Any distributed algorithm, deterministic or randomized, that computes a $\kappa$-approximately budget-balanced, GSP multicast cost-sharing mechanism, where $\kappa \leq \sqrt{2}-\epsilon$ for some fixed $\epsilon>0$, must send $\Omega\left(\frac{\log |P|}{\log \kappa}\right)$ bits of communication over linearly many links in the worst case. 
Proof: It is more convenient to work with an alternative representation of the input utility vectors. We use only a restricted set of the possible utility vectors $u$ and $v$ for the users located at nodes $B$ and $A$ respectively, where $(u, v)$ satisfies the following conditions:

- Let $\beta=3(\kappa+\delta)^{2} /\left(2-(\kappa+\delta)^{2}\right)$, where $\delta>0$ is an arbitrarily small constant only required to make the inequalities strict. Restrict the set of allowable utilities to $T=\left\{0, t_{\beta, 1}, \ldots, t_{\beta, r}\right\}$, where

$$
t_{\beta, i}=\frac{(\kappa+\delta) C}{2 \beta^{i+1}}
$$

- Each of $u$ and $v$ is (internally) sorted, i.e., $i<j \Rightarrow u_{i} \geq u_{j}$ and $v_{i} \geq v_{j}$. There is no restriction on the relationship between $u_{i}$ and $v_{j}$.

Consider node $B$. Define $n_{B}(q)$ to be the number of users at node $B$ who have utility at least $q$. Let $y=\left(y_{1}, y_{2}, \ldots, y_{r}\right)$, where $y_{i}=n_{B}\left(t_{\beta, i}\right)$. Similarly, let $n_{A}(q)$ be the number of users at $A$ with utility at least $q$; let $z=\left(z_{1}, z_{2}, \ldots, z_{r}\right)$, where $z_{i}=n_{A}\left(t_{\beta, i}\right)$.

For this class of utility profiles, there is a one-to-one mapping between values of $u$ and $y$. Because $u$ is sorted, the monotonically decreasing function $n_{B}(\cdot)$ completely defines the utility vector; $u_{1}$ must be the largest $q$ for which $n_{B}(q)>0$, and so on. Furthermore, by definition, there is a unique $y$ for any $u$. A similar correspondence holds for $v$ and $z$.

We first prove a useful lemma about approximately budget-balanced mechanisms on this class of instances.

Lemma 6 Let $M$ be a $\kappa$-approximately budget-balanced mechanism for the multicast cost sharing problem in Figure 2 that satisfies GSP. Let vectors $y, z$ be defined corresponding to the utility profiles $u$ and $v$, as described above. Then, $M$ satisfies the following two properties:

(i). If there is an $i$ such that $\left(y_{i}+z_{i}\right) \geq 2 \beta^{i+1}$, then mechanism $M$ will compute a non-empty receiver set on this instance.

(ii). If for all $i$ we have $\left(y_{i}+z_{i}\right) \leq\left(3 \beta^{i}+\beta^{i+1}\right)$, then mechanism $M$ will compute an empty receiver set on this instance.

Proof: (i): Observe that with a suitable ordering of the players, the conditions of Lemma 4 are satisfied if $\left(y_{i}+z_{i}\right) \geq 2 \beta^{i+1}$.

(ii): Assume that the receiver set is non-empty and that the conditions of Lemma 5 do not apply due to the presence of some $h$ such that $h \cdot \mu_{h} \geq C / \kappa$, where $\mu$ is the utility profile of $P$ sorted in decreasing order.

Let $\mu_{h}=t_{\beta, k}$ for some $k$. Since $h \leq y_{k}+z_{k}$, we note that $h \cdot \mu_{h} \leq\left(y_{k}+z_{k}\right) t_{\beta, k} \leq\left(3 \beta^{k}+\right.$ $\left.\beta^{k+1}\right) t_{\beta, k}=C /(\kappa+\delta)$. This violates the assumption that $h \cdot \mu_{h} \geq C / \kappa$.

We now use Lemma 6 to provide a reduction from the set disjointness problem as follows. Recall that node $A$ has a set $S_{1} \subseteq\{1,2, \ldots, r\}$ and node $B$ has another set $S_{2} \subseteq\{1,2, \ldots, r\}$. We must 
make sure that, if $S_{1} \cap S_{2} \neq \emptyset$, there is a set of receivers who can share $\kappa C$, and, if $S_{1} \cap S_{2}=\emptyset$, there is no set of receivers who can share even $C / \kappa$. For this, we construct the vectors $y$ and $z$ using the rules:

$$
\begin{aligned}
y_{i} & =\left\lceil\beta^{i+1}\right\rceil \quad \text { if } i \in S_{2} \\
y_{i} & =\left\lceil\beta^{i}\right\rceil \quad \text { otherwise } \\
z_{i} & =\left\lceil\beta^{i+1}\right\rceil \quad \text { if } i \in S_{1} \\
z_{i} & =\left\lceil\beta^{i}\right\rceil \quad \text { otherwise }
\end{aligned}
$$

These are valid input vectors, because $y_{i} \leq y_{i+1}$ and similarly for $z$. If $i \in S_{1} \cap S_{2}$, then $y_{i}+z_{i} \geq 2 \beta^{i+1}$, and so there is transmission. If $S_{1}$ and $S_{2}$ are disjoint, then, for all $i$,

$$
y_{i}+z_{i}<\beta^{i}+\beta^{i+1}+2<3 \beta^{i}+\beta^{i+1}
$$

where the 2 arises because of the ceiling terms.

This means that, in the target instance,

$$
\left(y_{i}+z_{i}\right) t_{\beta, i}<\frac{C}{\kappa},
$$

and consequently there is no transmission.

Thus, we can use this $\kappa$-approximate mechanism to solve the set-disjointness problem, and this implies that the mechanism must use $\Omega(r)$ bits of communication, where

$$
r=\Theta\left(\frac{\log p}{\log \beta}\right)=\Theta\left(\frac{\log p}{2 \log (\kappa+\delta)-\log \left(2-(\kappa+\delta)^{2}\right)}\right) .
$$

If we require $\kappa$ to be upper-bounded away from $\sqrt{2}$, then the right-hand side is $\Theta\left(\frac{\log p}{\log \kappa}\right)$. Thus, the statement of the theorem follows.

We note that this lower bound applies to the approximate mechanisms described in $[\mathrm{AFK}+02]$ and [JV01]. The mechanisms SF and SSF described in [AFK+02] provide the best known corresponding upper bound: They require $\Omega\left(h \cdot \frac{\log p}{\log \kappa}\right)$ utility values to be communicated on each link to achieve $\kappa$-approximate budget balance, where $h$ is the height of the multicast tree $T(P)$.

\section{An impossibility result for approximate budget-balance and ap- proximate efficiency}

As stated in Section 1, it is a classical result in game theory that no strategyproof cost-sharing mechanism can be both budget-balanced and efficient [GL79, Rob79]. We now consider whether this fundamental impossibility result holds when the budget-balance and efficiency considerations are replaced by their approximate counterparts. In this section, we do not assume that the costsharing mechanisms have the SYM property; the impossibility result that we present here does not 


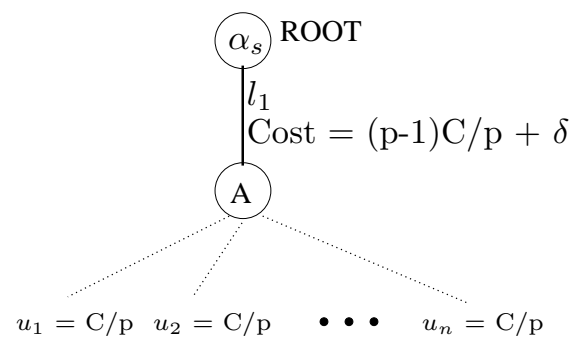

Figure 3: An example of a multicast tree that fails to achieve approximate efficiency and approximate budget-balance.

require this assumption. Furthermore, this result only requires the mechanism to be strategyproof, not GSP as in Section 3.

We first review the definition of the MC mechanism, which was shown by Moulin and Shenker [MS01] to be the only efficient mechanism that satisfies VP, NPT, and CS. Given an input utility profile $u$, the $\mathrm{MC}$ receiver set is the unique largest efficient set of users. To compute it, as shown in [FPS01], one recursively computes the welfare (also known as net worth or efficiency) of each node $\beta \in N$ :

$$
W(\beta)=\left(\sum_{\substack{\gamma \in C h(\beta) \\ W(\gamma) \geq 0}} W(\gamma)\right)-c(l)+\sum_{i \in \operatorname{Res}(\beta)} u_{i},
$$

where $C h(\beta)$ is the set of children of $\beta$ in the tree, $\operatorname{Res}(\beta)$ is the set of users resident at $\beta$, and $c(l)$ is the cost of the link connecting $\beta$ to its parent node. Then, the largest efficient set $R(u)$ is the set of all users $i$ such that every node on the path from $i$ to the root $\alpha_{s}$ has nonnegative welfare. The total efficiency is $\mathrm{N} W(R(u))=W\left(\alpha_{s}\right)$.

Another way to view this is as follows: The algorithm partitions the universal tree $T(P)$ into a forest $F(u)=\left\{T_{1}(u), T_{2}(u), \ldots, T_{k}(u)\right\}$. A link from $T(P)$ is included in the forest if and only if the child node has nonnegative welfare. $R(u)$ is then the set of users at nodes in the subtree $T_{1}(u)$ containing the root.

Once $F(u)$ has been computed, for each user $i$, define $X(i, u)$ to be the node with minimum welfare value in the path from $i$ to its root in its partition. Then, the cost share $x_{i}(u)$ of user $i$ is defined as

$$
\begin{array}{lll}
x_{i}(u)=\max \left(0, u_{i}-W(X(i, u))\right) & & \forall i \in R(u) \\
x_{i}(u)= & 0 & \forall i \notin R(u)
\end{array}
$$

If multiple nodes on the path have the same welfare value, we let $X(i, u)$ be the one nearest to $i$.

By a $\gamma$-approximately efficient mechanism, where $0<\gamma<1$, we mean one that always achieves total efficiency that is at least $\gamma$ times the total efficiency achieved by MC.

We can ask whether there is any strategyproof mechanism that satisfies the basic requirements of NPT, VP, and CS and is both approximately efficient and approximately budget-balanced. We 
now show that this is impossible, using the following approach: We construct a family of multicast trees and utility profiles for which any approximately efficient mechanism must transmit to all users. We show that the strategyproofness condition and the VP condition together place an upper bound on the revenue collected in these instances. This upper bound is less than that required for even approximate budget balance, and hence we have our negative result.

Consider the tree in Figure 3. There are $p$ users, each with utility $C / p$ resident at a node A that is separated from the root node by a link of cost $((p-1) C / p)+\delta$. It is easy to see that this instance of multicast cost-sharing displays the following properties.

Property 1 Any $\gamma$-approximately efficient mechanism must transmit to all $p$ users if $0<\delta<C / p$.

Property 2 Any $\gamma$-approximately efficient mechanism must transmit to all $p$ users even if one user, say $i$, lowers his utility to $\delta+\epsilon$, for any $\delta, \epsilon>0$.

Property 3 Any $\gamma$-approximately efficient, strategyproof mechanism that satisfies $V P$ assigns to each user a cost share of at most $\delta$.

If the cost share $x_{i}(u)$ were greater than $\delta$, the user $i$ could strategize by claiming that his utility was $v_{i}=\delta+\epsilon<x_{i}(u)$. By VP and the requirement of $\gamma$-approximate efficiency, the mechanism would need to include user $i$ and assign him a cost share $x_{i}\left(\left.u\right|^{i} v_{i}\right) \leq v_{i}<x_{i}(u)$, which would imply a violation of strategyproofness.

Therefore, the revenue collected by a strategyproof mechanism that achieves $\gamma$-approximate efficiency is bounded from above by $p \delta$.

Property 4 A $\gamma$-approximately efficient, strategyproof mechanism cannot be $\kappa$-approximately budgetbalanced if $\delta<(C(p-1)) /(p(\kappa p-1))$.

In summary, we have:

Theorem 4 A strategyproof mechanism for multicast cost sharing that satisfies the basic requirements of NPT, VP, and CS cannot achieve both $\gamma$-approximate efficiency and $\kappa$-approximate budgetbalance for any pair of constants $\kappa$ and $\gamma$.

\section{Conclusions and open problems}

As we explained in Section 1, one central challenge in the study of algorithmic mechanism design is the search for hard problems. A problem may be considered to be a "canonical hard problem" if it cannot be solved in a manner that satisfies both the incentive-compatibility and the computational-tractability requirements but becomes easy if one of these two requirements is dropped. Our results show that, under the basic requirements of NPT, VP, CS, and SYM, exact computation of budget-balanced multicast cost shares is such a problem. In this context, a 
problem is considered computationally tractable if it can be solved by a distributed algorithm with low network complexity; a solution is considered incentive compatible if it is group-strategyproof. Theorem 2 shows that the problem is hard if both requirements are to be satisfied simultaneously. If the computational-tractability requirement is dropped, the problem is easy: Just use the SH mechanism and compute it using the natural (centralized) polynomial-time algorithm explained in Section 2 above. If the incentive-compatibility requirement is dropped, it is easy as well: Just do one bottom-up pass of $T(P)$, computing $U=\sum_{i \in P} u_{i}$ and $C=\sum_{l \in L} c(l)$. If $C>U$, no one receives the transmission, and the mechanism does one top-down pass to inform all members of $P$ that this is the outcome; if $C \leq U$, everyone receives the transmission, and the mechanism does one top-down pass to communicate the cost share $\left(u_{i} \cdot C\right) / U$ to user $i$, for all $i \in P$.

The most important general direction for future work is the search for more good distributed algorithmic mechanisms and for more canonical hard problems. Additional general open questions in DAMD can be found in [FS02].

More specifically, we ask whether the lower bound in Section 3 is tight. The "scaled stepfunction" (SSF) mechanism considered in $[\mathrm{AFK}+02]$ gives some hope that it is, but the bounds on budget-imbalance and efficiency-loss for SSF are not constant factors. Another interesting open question is to ask if the communication lower bounds extend to strategyproof (but not necessarily group-strategyproof) budget-balanced mechanisms.

\section{References}

[AFK+02] Archer, A., Feigenbaum, J., Krishnamurthy, A., Sami, R., and Shenker, S. (2002). "Approximation and Collusion in Multicast Cost Sharing," submitted. Available in preprint form at http://www.cs.yale.edu/homes/jf/AFKSS.ps.

[AR02] Adler, M. and Rubenstein, D. (2002). "Pricing Multicast in More Practical Network Models," in Proceedings of the 13th Symposium on Discrete Algorithms, pp. 981-990, ACM Press/SIAM, New York/Philadelphia.

[BFC93] Ballardie, A., Francis, P., and Crowcroft, J. (1993). "Core Based Trees (CBT)," in Proceedings of SIGCOMM '93, pp. 85-95, ACM Press, New York.

[DEF+96] Deering, S., Estrin, D., Farinacci, D., Jacobson, V., Liu, C., and Wei, L. (1996). "The PIM architecture for wide-area multicast routing," ACM/IEEE Transactions on Networking 4, pp. 153-162.

[DR89] Dutta, B. and Ray, D. (1989). "A concept of egalitarianism under participation constraints," Econometrica 57, pp. 615-635. 
[FGH+02] Fiat, A., Goldberg, A., Hartline, J., and Karlin, A. (2002). "Competitive Generalized Auctions," in Proceedings of the 34th Symposium on the Theory of Computing, pp. 72-81, ACM Press, New York.

[FKS+02] Feigenbaum, J., Krishnamurthy, A., Sami, R., and Shenker, S. (2002). "Hardness Results for Multicast Cost Sharing (Extended Abstract)," in Proceedings of the 22nd Conference on Foundations of Software Technology and Theoretical Computer Science, Lecture Notes in Computer Science, Volume 2556, pp. 133-144, Springer, Berlin.

[FNY89] Ferguson, D., Nikolaou, C., and Yemini, Y. (1989). "An economy for flow control in computer networks," in Proceedings of the 8th Infocom, pp. 100-118, IEEE Computer Society Press, Los Alamitos.

[FPS01] Feigenbaum, J., Papadimitriou, C., and Shenker, S. (2001). "Sharing the cost of multicast transmissions," Journal of Computer and System Sciences 63, pp. 21-41.

[FS02] Feigenbaum, J. and Shenker, S. (2002). "Distributed Algorithmic Mechanism Design: Recent Results and Future Directions," in Proceedings of the 6th International Workshop on Discrete Algorithms and Methods for Mobile Computing and Communication, pp. 1-13, ACM Press, New York.

[FS97] Friedman, E., and Shenker, S. (1997). "Learning and Implementation in the Internet," preprint. Available at http://www.icir.org/shenker/decent.ps

[GL79] Green, J. and Laffont, J-J. (1979). Incentives in Public Decision Making, North Holland, Amsterdam.

[HA88] Hsiao, M.-T. and Lazar, A. (1988). "A game theoretic approach to decentralized flow control of Markovian queueing networks," in Proceedings of Performance '87, pp. 5574, North-Holland, Amsterdam.

[HC99] Holbrook, H. and Cheriton, D. (1999). "IP multicast channels: Express support for large-scale single-source applications," in Proceedings of SIGCOMM '99, pp. 65-78, ACM Press, New York.

[HSE97] Herzog, S., Shenker, S., and Estrin, D. (1997). "Sharing the 'cost' of multicast trees: An axiomatic analysis," IEEE/ACM Transactions on Networking 5, pp. 847-860.

[J01] Jackson, M. (2001). "A Crash Course in Implementation Theory," Social Choice and Welfare 18, pp. 655-708.

[JV01] Jain, K. and Vazirani, V. (2001). "Applications of approximation to cooperative games," in Proceedings of the 33rd Symposium on the Theory of Computing, pp. 364372, ACM Press, New York. 
[KLO95] Korilis, Y., Lazar, A., and Orda, A. (1995). "Architecting noncooperative networks," Journal on Selected Areas in Communications 13, 1241-1251.

[KN97] Kushilevitz, E. and Nisan, N. (1997). Communication Complexity, Cambridge University Press, Cambridge.

[KS89] Kurose, J. F. and Simha, R. (1989). "A microeconomic approach to optimal resource allocation in distributed computer systems," IEEE Transactions on Computers $\mathbf{3 8}$, 705-717.

[M99] Moulin, H. (1999). "Incremental cost sharing: characterization by strategyproofness," Social Choice and Welfare 16, pp. 279-320.

[MS01] Moulin, H. and Shenker, S. (2001). "Strategyproof Sharing of Submodular Costs: Budget Balance Versus Efficiency," Economic Theory 18, pp. 511-533.

[MT02] Mitchell, J. and Teague, V. (2002). Private communication.

[MT99] Monderer, D. and Tennenholtz, M. (1999). "Distributed Games: From Mechanisms to Protocols," in Proceedings of the Sixteenth National Conference on Artificial Intelligence and Eleventh Conference on Innovative Applications of Artificial Intelligence (AAAI/IAAI' '99), pp. 32-37, AAAI/MIT Press.

[NR00] Nisan, N. and Ronen, A. (2000). "Computationally feasible VCG mechanisms," in Proceedings of the 2nd Conference on Electronic Commerce (EC-00), pp. 242-252, ACM Press, New York.

[NR01] Nisan, N. and Ronen, A. (2001). "Algorithmic mechanism design," Games and Economic Behavior 35, pp. 166-196.

[NS02] Nisan, S. and Segal, I. (2002). "The Communication Complexity of Efficient Allocation Problems," (working paper).

[PLB+99] Perlman, R., Lee, C.-Y., Ballardie, A., Crowcroft., J., Wang, Z., Maufer, T., Diot, C., and Green, M. (1999). "Simple multicast: A design for simple low-overhead multicast," IETF Internet Draft (Work in Progress).

[Rob79] Roberts, K. (1979). "The Characterization of Implementable Choice Rules," in JJ. Laffont, editor, Aggregation and Revelation of Preferences, pp. 321-348, North-Holland, Amsterdam.

[S88] Sanders, B. (1988). "An incentive compatible flow control algorithm for rate allocation in computer networks," IEEE Transactions on Computers 37, 1067-1072. 
[S90] Shenker, S. (1990). "Efficient network allocations with selfish users," in Proceedings of Performance '90, pp. 279-285, North-Holland, Amsterdam.

[S95] Shenker, S. (1995). "Making greed work in networks," ACM/IEEE Transactions on Networking 3, 819-831. 